\title{
A simple cornea model for the tribological performance assessment of the lubricating eye drops
}

\author{
Hakan Göcerler and Simon Medina \\ Department of Tribology, TU Wien Institute of Engineering Design and Product Development, Wien, Austria \\ Michael Adler and Fosef Brenner \\ AC2T Research GmbH, Wiener Neustadt, Austria, and \\ Andreas Tadler, Michael Nagl and Christine Hohenadl \\ Croma-Pharma GmbH, Leobendorf, Austria
}

\begin{abstract}
Purpose - Dry eye syndrome is one of the most common reasons for eye-related discomfort which, without treatment, in some cases may even lead to corneal damage. Blinking, baseline and reflex lachrymation and drainage compromise the topical application of therapeutics demanding repeated, often hourly applications of common lubricants. In contrast, topically administered chitosan-N-acetylcysteine-based eye drops were reported to sustain on the ocular surface for more than $24 \mathrm{~h}$. The thiolated biopolymer can interact with the corneal mucin layer thereby forming covalent disulphide bridges, which may contribute to extended residence times.

Design/methodology/approach - In this study, the tribological characteristics of four different lubricants including hyaluronic acid and chitosan$\mathrm{N}$-acetylcysteine containing commercially available eye drops were investigated. For this purpose, a representative test setup was developed, which mimics the contact between the cornea and the eyelid wiper. Gels with different elastic properties coated with a mucin layer were used as a substrate to mimic the corneal surface. Tests were conducted with a micro-tribometer, and friction values were recorded. Contact zones were characterized by $\mathrm{X}$-ray photoelectron spectroscopy to investigate wear and thiol bonding on the surface.

Findings - Results revealed the lowest average coefficient of friction values for chitosan-N-acetylcysteine-based eye drops and substrate dependence of the test setup.

Originality/value - In this study, the authors introduced an in vitro system to test different types of eye drops so that chemical interaction with the mucin layer can be observed. These interactions change the tribological performance significantly and must be considered to have results relevant to the actual application.
\end{abstract}

Keywords Biotribology, Biomimetic, Cornea, Eye drops, Tribometry

Paper type Research paper

\section{Introduction}

Dry eye syndrome (DES) is a multifactorial disease of the ocular surface characterized by a loss of homeostasis of the tear film. Eventually the disease makes the eye vulnerable to external stimuli and inflammation (Thulasi and Djalilian, 2017; Ludwig, 2005). A new treatment approach involves the use of chitosan-N-acetylcysteine-based eye drops (C-NAC$\mathrm{BED})$. These eye drops lead to the formation of a long-lasting, mucoadhesive protective layer on the surface of the cornea (Fischak et al., 2017). C-NAC-BEDs were accordingly approved in Europe as a class III medical device for alleviation of DES symptoms.

Blinking, baseline and reflex lachrymation and drainage compromise the topical application of therapeutics, demanding

The current issue and full text archive of this journal is available on Emerald Insight at: https://www.emerald.com/insight/0036-8792.htm

Industrial Lubrication and Tribology

73/10 (2021) 1294-1301

Emerald Publishing Limited [ISSN 0036-8792] [DOI 10.1108/ILT-08-2021-0296] repeated, often hourly applications of common lubricants. Topically administered C-NAC-BED, however, were reported to sustain on the ocular surface for more than $24 \mathrm{~h}$. This indicates distinct tribological properties of the thiolated chitosan functioning as a lubricant between the eyelid and the cornea, which have not yet been investigated.

To evaluate the mechano-chemical behavior of chitosan-Nacetylcysteine, it was crucial to define the mechanical properties of the model substrate to be used in tribological tests.

(C) Hakan Göcerler, Simon Medina, Michael Adler, Josef Brenner, Andreas Tadler, Michael Nagl and Christine Hohenadl. Published by Emerald Publishing Limited. This article is published under the Creative Commons Attribution (CC BY 4.0) licence. Anyone may reproduce, distribute, translate and create derivative works of this article (for both commercial and non-commercial purposes), subject to full attribution to the original publication and authors. The full terms of this licence may be seen at http://creativecommons.org/licences/by/4.0/legalcode

Conflicts of interest: Andreas Tadler, Michael Nagl and Christine Hohenadl are employees of Croma-Pharma $\mathrm{GmbH}$.

Received 4 August 2021

Revised 24 September 2021

Accepted 4 October 2021 
The first step was to create a substrate mimicking the ocular mucins, which structure themselves in a gradient from a solute in the aqueous tear film to a gel on the corneal surface, with accurate Young's modulus. Young's modulus (elastic modulus) is a material property and is a measure of the ability of a material to withstand changes in length when under lengthwise tension or compression. Reported values for Young's modulus of the cornea exhibit a considerable variation due to different measurement techniques. A simulation model of an eyeball based on finite element analysis suggested $20 \mathrm{kPa}$ for retina (Wu et al., 2013), and biomechanical studies of a fluid-filled spherical shell model with Scheimpflug imaging calculated values between $54-359 \mathrm{kPa}$ for the cornea (Shih et al., 2017). Studying compliance profiles of the human cornea using atomic force microscopy (AFM) focused on different layers of the cornea and obtained values of $7.5 \pm 4.2 \mathrm{kPa}$ (anterior basement membrane), $109.8 \pm 13.2 \mathrm{kPa}$ (Bowman's layer), $33.1 \pm 6.1 \mathrm{kPa}$ (anterior stroma) and $50 \pm 17.8 \mathrm{kPa}$ (Descemet's membrane) (Last et al., 2012). In vivo determinations of Young's modulus for the human corneal basement membranes using AFM also reveal values of $25 \pm 7 \mathrm{kPa}$ (Last et al., 2009). The corneal epithelial cell response to the substrate stiffness was underlined by using polyacrylamide substrates with different elastic moduli (Molladavoodi et al., 2014). Also, it is observed that Young's modulus of the anterior corneal stroma is altered with the initiation of oedema and inflammation (Raghunatan et al., 2017). In this study, we designed two types of gels with the same composition but different Young's modulus values $(18 \mathrm{kPa}$ and $36 \mathrm{kPa}$ ) to simulate the mucin layer and to establish the interaction of the mucin layer and chitosan-Nacetylcysteine. By this means we should be able to observe differences in the coefficient of friction (COF) values, not only due to different solutions but also due to different grades of stiffness of the substrate.
Another critical parameter for tribological systems is the load to be applied. As seen with Young's modulus values, also the load applied onto the system within the literature varies within a broad range. Three possible models of eyelid contact with the pressure cell were introduced for eyelid pressure calculations: the eyelid contacts with the whole cell [Figure 1(A)], contact is over the imprint width determined by micro paper imprint [Figure 1(B)] and finally contact over Marx's line width determined by lissamine-green staining and digital imaging [Figure 1(C)] (Shaw et al., 2010). In a whole cell contact the calculated eyelid pressure was as small as $0.3 \mathrm{kPa}$, whereas in Marx's line contact, this value was measured to be $18.6 \mathrm{kPa}$. We presume in an eye with DES, a whole cell contact could be observed rarely due to lack of tear fluid; thus, the maximum eyelid pressure should be considered for modelling a test system so that a contact system described in (Raghunatan et al., 2017) is preferred [Figure $1(\mathrm{C})$ ]. $12 \mathrm{kPa}$ was calculated for the test system designed to measure COF values for murine cornea in vivo (Dunn et al., 2013a). Normal load values between $10-50 \mathrm{mN}$ were applied to observe COF values for soft contact lenses (Rennie et al., 2005). Normal loading forces of $22.7 \mathrm{mN}$ and $81.4 \mathrm{mN}$ were applied, to observe differences between silicone hydrogel versus conventional hydrogel contact lenses (Ngai et al., 2005). Finally, Aston Biomaterials Research Unit (ABRU) used assay standards with a load of $30 \mathrm{mN}$ for tests on Hypotears ${ }^{\mathrm{TM}}$ (Mann and Tighe, 2006). In our study, $20 \mathrm{mN}$ normal load was applied to achieve $12 \mathrm{kPa}$ pressure on the substrate, which is matching with eyelid pressure calculations and tests conducted in the literature. Of course, it should be expected that this value varies in situ due to personal health condition, age and gender of people (Rico-del-Viejo et al., 2017). In this work, we consider most common and relatively high pressure values of Marx's line to evaluate the limits of CNAC-BED.

Figure 1 Three possible models of eyelid contact with the pressure cell were introduced for eyelid pressure calculations (Shaw et al., 2010)

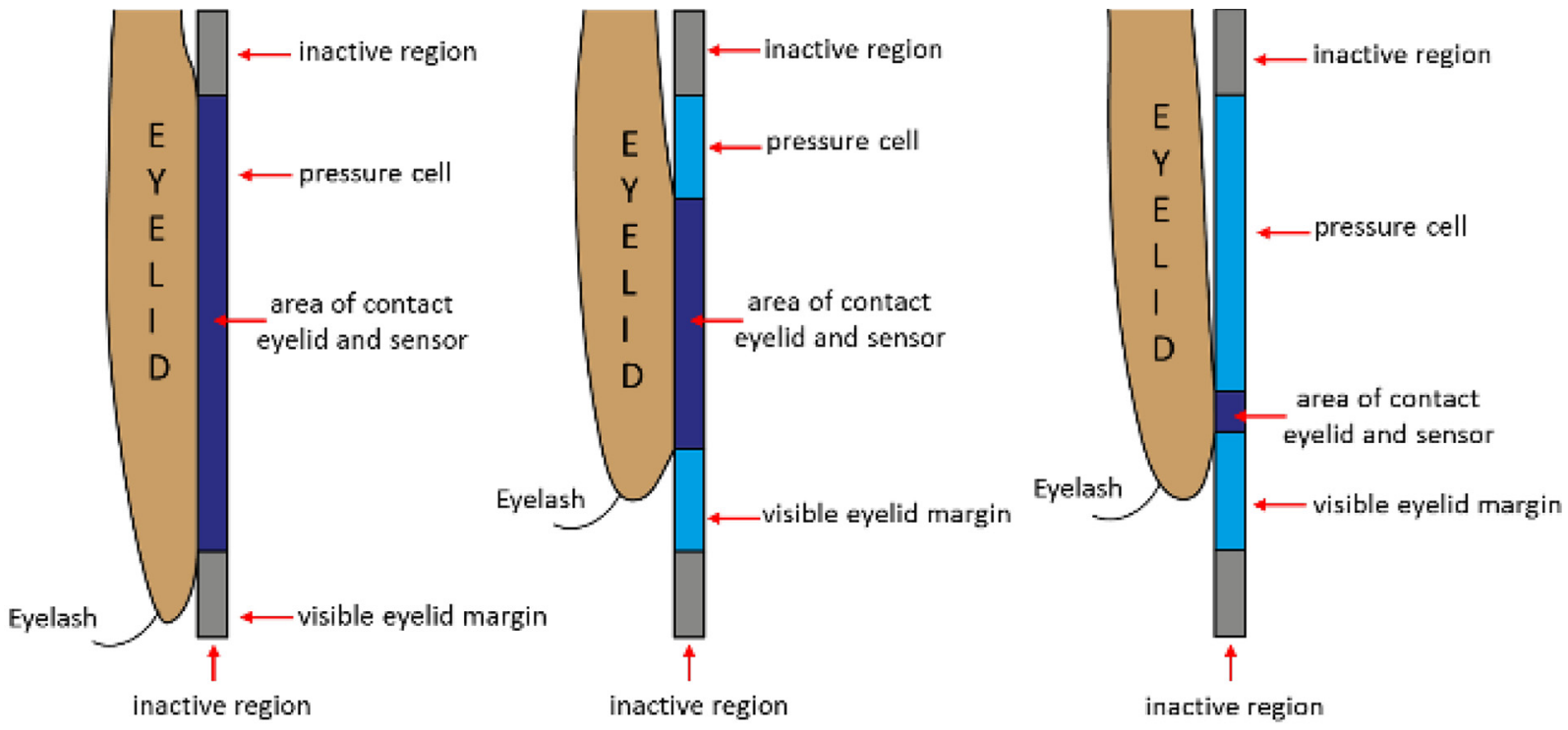

A) "whole cell" eyelid pressure

B) "imprint width" eyelid pressure

C) "Marx's line" eyelid pressure 
Even though the maximum velocity during a blink was reported as $300 \mathrm{~mm} / \mathrm{s}$ (closing) and $100 \mathrm{~mm} / \mathrm{s}$ (opening) (Jones et al., 2008), the standard condition used at ABRU was $0.5 \mathrm{~mm} / \mathrm{s}$. In our study, a velocity of $2 \mathrm{~mm} / \mathrm{s}$ was chosen to avoid additional vibrations and provide a stable translational movement for the micro-tribometer. We selected a stroke of $2 \mathrm{~mm}$ to observe the contact zone and to evaluate the tribological performance of the lubricative solutions.

Finally, to observe the described mucoadhesive properties of C-NAC-BED, chemical analysis was conducted using X-ray photoelectron spectroscopy (XPS). Bonded chitosan-Nacetylcysteine layers were investigated in the wear track following the tests.

\section{Experimental details}

\subsection{Specimen preparation}

The base body for the following tribological measurements consisted of petri dishes filled with stabilized (glutaraldehyde crosslinked) gelatine (platinum grade) in layers of different thickness ranging from $0.5 \mathrm{~mm}$ (generally higher hardness due to collapsing) to $3 \mathrm{~mm}$ (lower hardness). The gelatine surfaces were coated with a mucin layer (porcine stomach Type III, bound sialic acid $0.5-1.5 \%$ ). After coating, the gelatine plate was washed five times with water for injection, and the integrity of the mucin layer was tested by alcian-blue staining.

Four different solutions were used as lubricants for the tribological tests, namely saline $(0.9 \% \mathrm{NaCl}$ w/w, denoted below as " $\mathrm{NaCl}$ "), chitosan-hydrochloride $(1 \mathrm{mg} / \mathrm{mL})$ in a physiological buffer system (denoted as "Placebo"), and the commercially available eye drops Lacrimera ${ }^{\circledR}$, Croma-Pharma $\mathrm{GmbH}$. ("CNAC-BED", based on chitosan-N-acetylcysteine) and Hylo Comod®, Ursapharm GmbH. ("H-BED", based on hyaluronate). Basically "Placebo" is a solution of non-derivatized chitosan, identically buffered as C-NAC-BED. Prior to testing, each plate was pre-conditioned by soaking in a physiological sodium chloride solution. Two drops of each solution were applied onto the test zones, given 5 min of soaking time, and tests were conducted at room temperature with a $45 \%$ humidity level.

\subsubsection{Determination of Young's modulus}

Identification of Young's modulus $(E)$ is crucial to evaluate contact conditions in tribological tests. Macro-indentation tests were conducted to calculate the $\mathrm{E}$ value of the gelatine hydrogel substrates (Figure 2) produced for the designed tribological system. In such a test, the surface is dented by a probe of defined shape while the required force is measured with a spring. The tribometer was also used as an indenter, using vertical instead of lateral movement. A $5 \mathrm{~mm}$ alumina oxide ceramic ball, identical to those used for tribological experiments, was used as an indentation probe (Figure 3). The indentation mode, based on the identified penetration depth and indentation speed, was used. A maximum penetration depth of $0.5 \mathrm{~mm}$ was selected to remain in the elastic range of the gels and to ensure that no rupture occurs in the mucin layer. The indentation speed was $0.01 \mathrm{~mm} / \mathrm{s}$, to avoid sudden changes due to the visco-elastic behavior of the gels.

The micro-tribometer (TETRA BASALT®-N2) recorded the deflection of the spring during the approach. The calibrated system then translated this data into the normal load values [Figures 4 and 5(a)]. Black dots on Figure 5(b) show the locations of the indentation tests (each plate once on each location); the Young's modulus was obtained by fitting the indentation force vs. indentation depth of the plate with an overlay of the theoretical force based on the Hertz model, as shown in equation (1) (Thomasy et al., 2014) (Figure 4, dashed lines):

Equation (1): Force at indentation test

$$
F=\frac{4}{3} * \frac{E}{\left(1-v^{2}\right)} * \delta^{3 / 2} * R^{1 / 2}
$$

where $\mathrm{F}$ is the force applied by the indenter, $\mathrm{E}$ is Young's modulus, $v$ is Poisson's ratio, $\mathrm{d}$ is the indentation depth and $\mathrm{R}$ is the radius of the tip. Poisson's ratio of 0.49 is selected for evaluating material parameters of both the cornea and sclera in a numerical model (Ś ródka, 2011).

Elasticity modulus for the soft (not collapsed) and hard (collapsed) gel samples was calculated by iteration using the initial value given by Equation (1) as $18 \mathrm{kPa}$ and $36 \mathrm{kPa}$, respectively.

\subsection{Setup of tribological test}

The tribometer used for indentation tests [Figure 5(a)] was used in translational mode for tribological tests. Similar tribometer installations have been used in ocular bio-tribology

Figure 2 Sample configuration, supporting gel layer poured into petri dish and coated with mucin layer

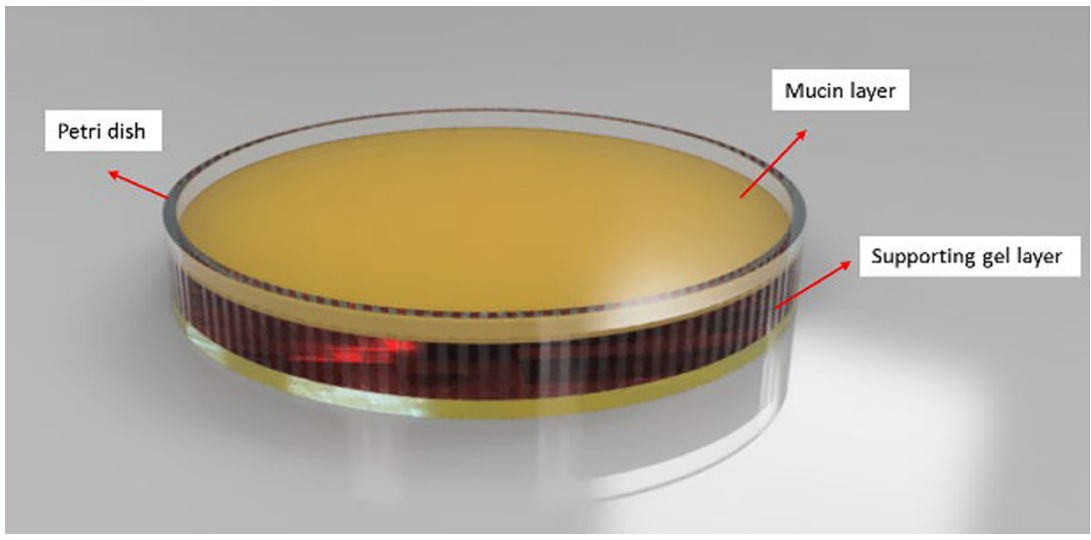


Figure 3 Image captured during the indentation test

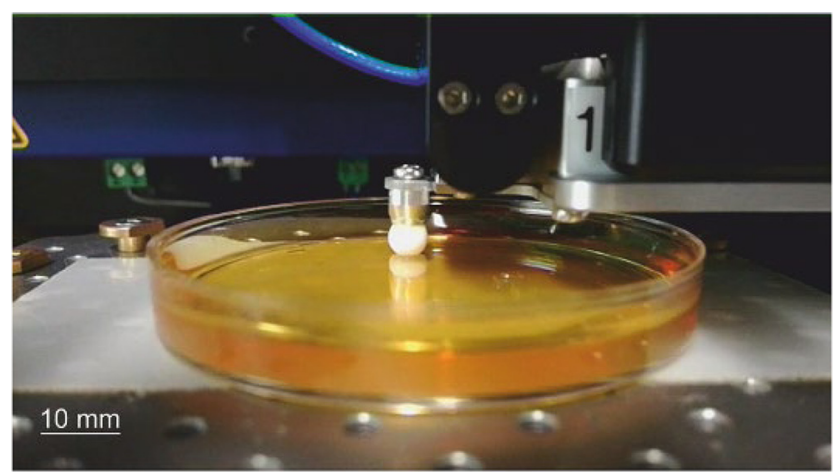

(Urueña et al., 2018; Samsom et al., 2015; Colter et al., 2017; Mollenhauer et al., 2006) for measurements of the friction force between biological base bodies and several counter bodies. Due to relatively low applied load values $(\mathrm{Fn} \leq 1 \mathrm{~N}$ ), tribometers mentioned in these installations are called micro-tribometers.

Modules and sensors were selected according to the loading conditions and movement of the eye-eyelid system, as described in literature (Dunn et al., 2013b; Sterner et al., 2017; Cobb et al., 2008; Tomala et al., 2013).

Tribological tests were conducted on two types of coated hydrogel plates (soft and hard) with a normal load of $20 \mathrm{mN}$, a reciprocal motion at a frequency of $2 \mathrm{~Hz}$ and a stroke of $2 \mathrm{~mm}$. All experiments were performed with 1000 cycles, on a total of eight different plates. Friction data is recorded via data acquisition software in TETRA BASALT $®-\mathrm{N} 2$ that captures 100 data points for each cycle and takes the average value discarding the acceleration and deceleration parts of the probe movement. Eventually the average of 8 tests for each specimen on each substrate was taken for establishing the data for the friction values represented in Figures 7 and 8.

\subsection{Surface chemistry determination X-ray photoelectron spectroscopy}

XPS measurements were performed on a Theta Probe ${ }^{\mathrm{TM}}$ (Thermo Fisher Scientific) to investigate the presence of disulphide bonds confirming the binding of mucin with CNAC or with itself and to document changes in surface chemistry due to tribological testing. A typical camera image inside the XPS chamber with the selected measurement position in a wear track (red lined area) is given in Figure 6 exemplarily for the sample "Placebo."

The X-ray source is a monochromatic $\mathrm{Al} \mathrm{K}$ (alpha) source at $1386.6 \mathrm{eV}$. The spectrometer is calibrated to $368.21 \mathrm{eV}$ binding energy (BE) of the Ag $3 \mathrm{~d} 5 / 2$ line for metallic silver and the linearity is corrected to BE of metallic $932.62 \mathrm{eV}$ for the $\mathrm{Cu} 2 \mathrm{p} 3 / 2$ line and $83.96 \mathrm{eV}$ and $\mathrm{Au} 4 \mathrm{f} 71 / 2$. Charge compensation is done using a flood gun for low-energy electrons and argon ions at $1 \mathrm{eV}$. The binding energy of the corrected spectra are corrected to the main hydrocarbon peak of C1s at $284.6 \mathrm{eV} \mathrm{BE}$.

The resolution of the X-ray spot was set to $50 \mu \mathrm{m}$ for all measurements. The survey spectra were recorded with a pass energy of $200 \mathrm{eV} \mathrm{BE}$ and the detail spectra with a pass energy of $50 \mathrm{eV}$. The base pressure in the analytical chamber was $<5^{*} 10^{-8} \mathrm{~Pa}$. Avantage v5.9915 (Thermo Fischer Scientific) was used to evaluate the XPS spectra. Peaks were fitted using a convolution of Lorentzian and Gaussian peak shapes. Binding states of elements detected were analyzed in reference to the NIST XPS database (NIST, 2012).

Figure 4 Force as function of displacement for soft (continuous light green) and hard (continuous light blue line) gelatine gels vs calculated value (CV) by iteration for each material (in dashed lines)

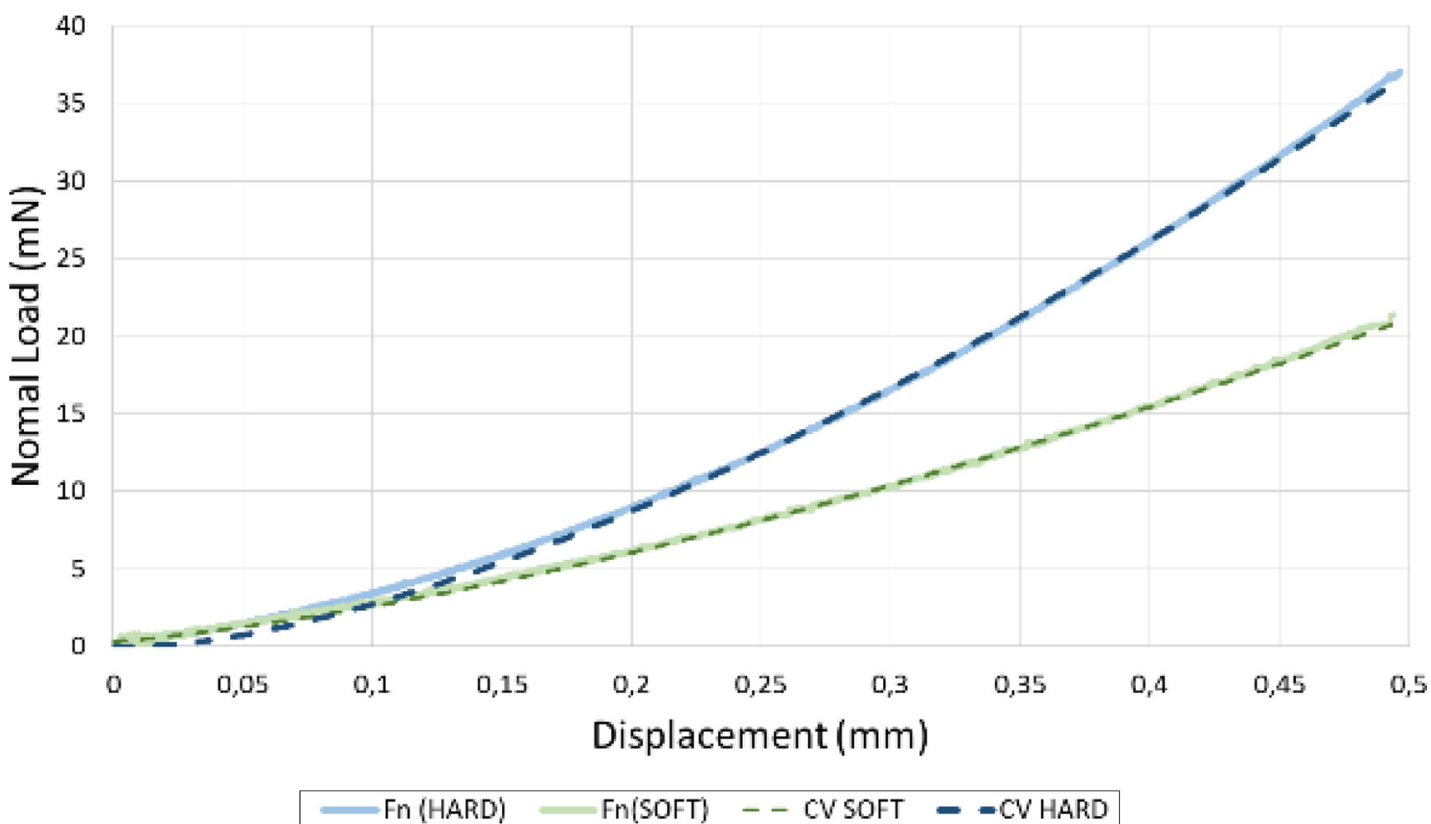


Figure 5 (a) Details of the capacitive sensor and ceramic probe attached on the spring of the tribometer and (b) Schematic presentation of a gelatine hydrogel plate with locations of applied solutions and performed tests (strokes)

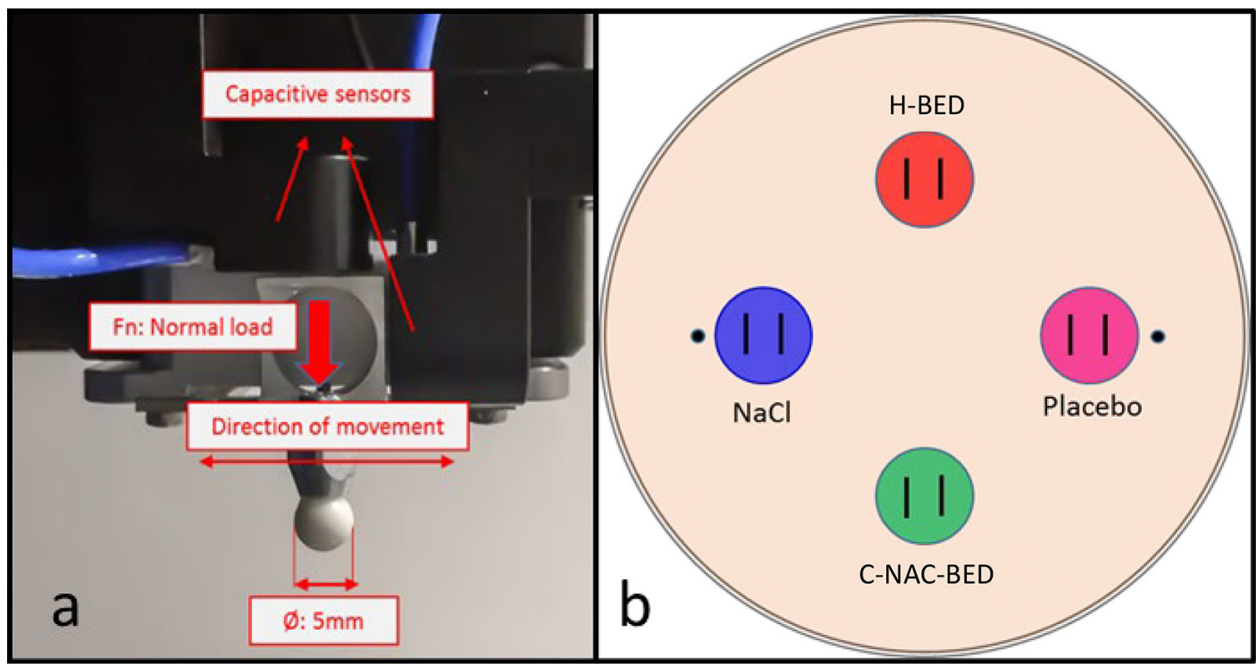

Notes: Dark dots represent positions of indentation tests

Figure 6 Camera image inside the XPS chamber to spot area of contact (red line)

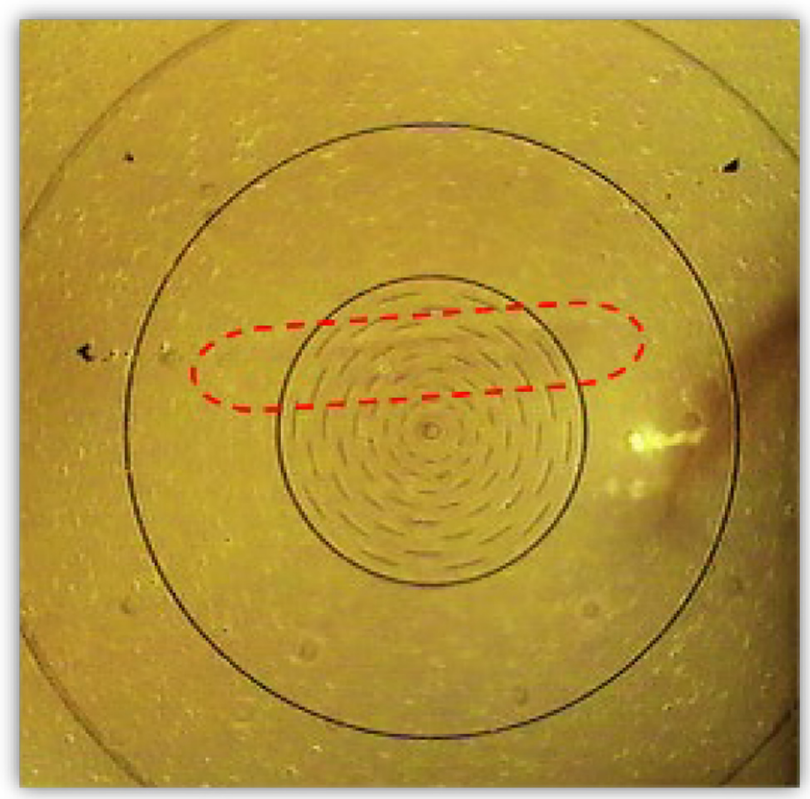

For the XPS analyses, samples were cut out with a scalpel to a size of approximately $5 \times 5 \mathrm{~mm}^{2}$. They were dried in four steps using ethanol of increasing concentration. The evacuation in the fast entry lock was performed for several days to maintain the vacuum performance in the analytical XPS chamber.

\section{Results and discussion}

\subsection{Micro-tribometer tests}

Tests were conducted in a rotational manner; each plate started with another solution and continued with the next solution counter clockwise [Figure $5(\mathrm{~b})$ ], to reduce the effect of drying for each solution. Results represent the average value of four repetitions for each sample. Average COF values calculated by averaging every data point for four tests per cycle show the same ranking for both soft and hard substrates. $\mathrm{COF}$ values for $\mathrm{C}-\mathrm{NAC}-\mathrm{BED}, \mathrm{H}-\mathrm{BED}, \mathrm{NaCl}$ and Placebo are 0.32, 0.35, 0.37 and 0.40, respectively, for hard hydrogels (Figure 7).

A slight increase in average COF was observed for soft hydrogel substrates. COF for C-NAC-BED increased to 0.37 , for $\mathrm{H}-\mathrm{BED}$ to $0.40, \mathrm{NaCl}$ to 0.45 and for the Placebo to 0.47 (Figure 8). The ranking among the test solutions did not change. Differences are possibly due to a larger area of interaction that is expected with the greater surface conformity of softer gels. Similar observation reported for the effect of dwell time in creeping elastic-plastic polymers (Malamut et al., 2009) and articular cartilages that was reinforced with tissueinterpenetrating polymers (Cooper et al., 2017). COF values of hard gels, more like a solid base body, stayed at a constant friction level, in contrast to soft gels, for which a slight steady increase was observed during the duration of the tribological tests. C-NAC-BED produced the lowest COF values in both configurations.

\subsection{X-ray photoelectron spectroscopy results}

On the reference surfaces outside the wear track, sulphur can be detected essentially on all samples at about $1 \%$, so that coating with mucin can be assumed. The sample "Placebo" has the highest content of sulphur, and the detailed analysis shows that it is present in oxidized form, mainly in the state $+4^{-}$ (Figure 9). BE of $167 \mathrm{eV}$ to $168 \mathrm{eV}$ are attributed to oxidized states, whereas S-S binding types are reported app. at BE of 163 to $165 \mathrm{eV}$ (NIST, 2012).

Sample C-NAC-BED shows a similar total S content outside the wear track, compared to reference surface as sample "Placebo." To a small extent, disulphide binding energy is 
Figure 7 COF results for hard hydrogel substrate samples

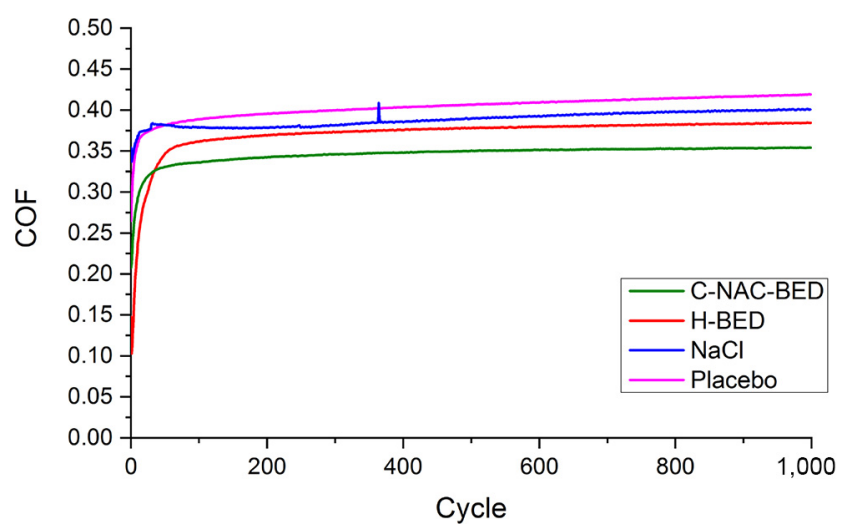

Figure 8 COF results for soft hydrogel substrate samples

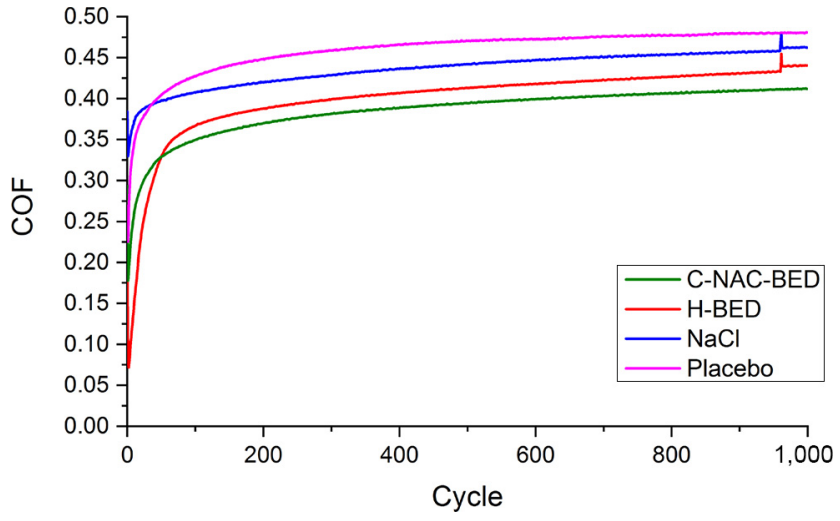

Figure 9 XPS S 2p outside the wear track (reference) of sample Placebo

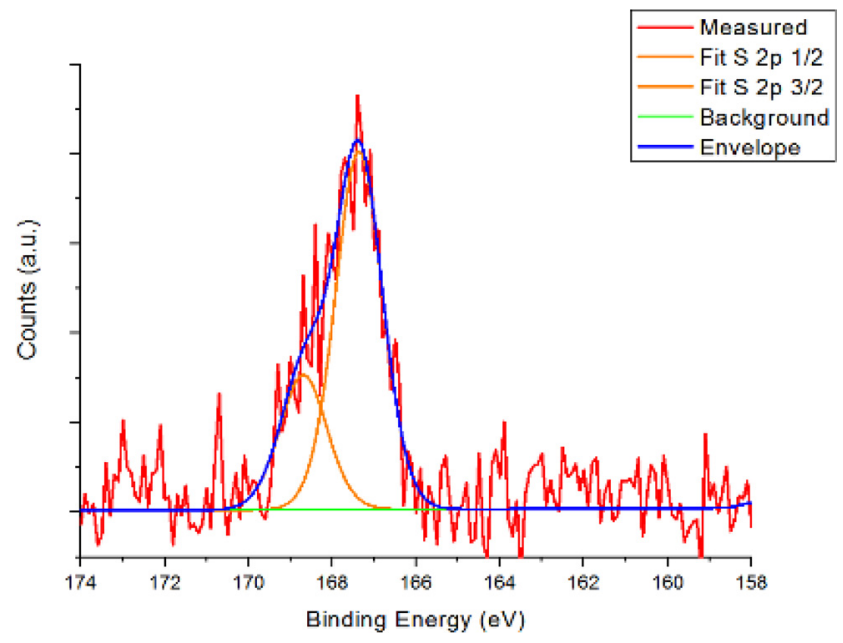

detectable (Figure 10), whereas most of the $S$ is present in oxidized states, mainly +4 . On sample H-BED, the lowest $S$ concentration is detected, with the disulphide value below the detection limit (Figure 11).
Figure 10 XPS S 2p spectrum of sample C-NAC-BED outside the wear track (reference) Presence of $S-S$ chemical state is evident through necessary peak fittings at BE $\sim 162$ to $164 \mathrm{eV}\left(\right.$ NIST, 2012) ${ }^{a}$

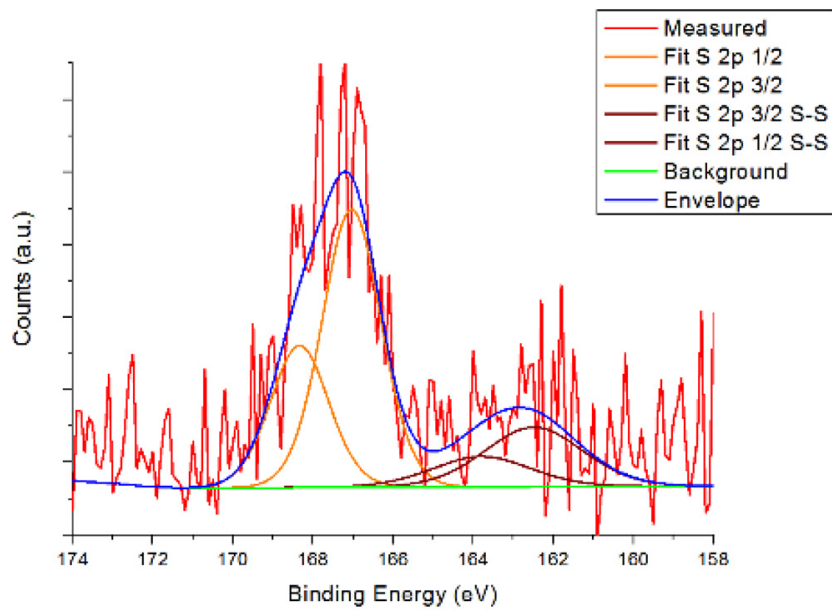

Note: ariginal spectra without applied correction for charge compensation is shown. A correction to $\mathrm{Cls}$ at reference $\mathrm{BE}$ at $184.6 \mathrm{eV}$ shifts the binding energies app. $1.5 \mathrm{eV}$ to higher $\mathrm{BE}$

Figure 11 XPS S 2p spectrum of sample HBED

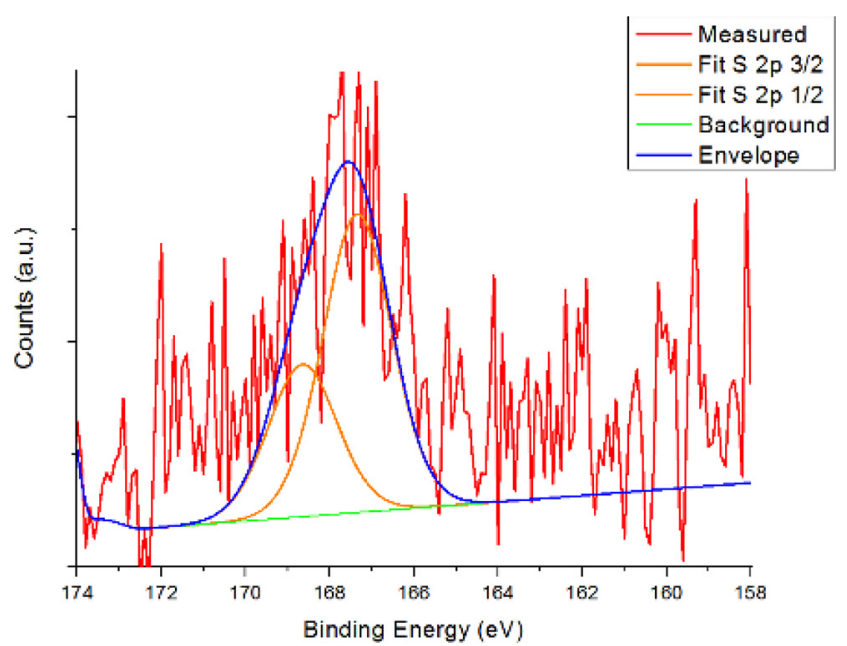

Table 1 summarizes the relative concentrations of the detected elements for each sample, for both worn and reference surfaces. A reduction of contaminations $(\mathrm{Si}, \mathrm{Ca})$ in the wear track is detectable due to the frictional load on all samples. The sample "Placebo" shows a slightly different behavior than "H-BED" and "C-NAC-BED" regarding the element composition for $\mathrm{N}$ and $\mathrm{O}$ in the wear track compared to the reference surface. While in the sample "Placebo" the $\mathrm{N}(\sim 10 \%)$ and $\mathrm{O}(\sim 32 \%)$ content do not vary noticeably, an increase in concentration in the wear tracks "H-BED" ( $\mathrm{N}$ from $7 \%$ to $14 \%$, O from $19 \%$ to $22 \%$ ) and "C-NAC-BED" ( $\mathrm{N}$ from $5 \%$ to $12 \%$, O from $16 \%$ to $22 \%$ ) samples is significantly detectable. The measurable increase of $\mathrm{N}$ and $\mathrm{O}$ can be attributed to the wear of the protective layer of the H-BED and C-NAC-BED, compared to the sample "Placebo" in which there is no activated protective 
Table 1 XPS Relative element concentration on the reference and the worn surface of samples "placebo," "H-BED" and "C-NAC-BED," respectively

\begin{tabular}{|c|c|c|c|c|c|c|c|c|}
\hline \multirow[b]{2}{*}{ Sample } & \multicolumn{3}{|c|}{ Relative elemental concentration (at $\%$ ) } & \multirow[b]{2}{*}{ C } & \multirow[b]{2}{*}{$\mathrm{N}$} & \multirow[b]{2}{*}{0} & \multirow[b]{2}{*}{ Si } & \multirow[b]{2}{*}{$\mathrm{Ca}$} \\
\hline & Position & $\mathbf{S}$ & S-chem states & & & & & \\
\hline Placebo & Reference & 1 & $\left(\mathrm{SO3/S04)^{2- }}\right.$ & 55 & 9 & 34 & $<1$ & n.d. \\
\hline Placebo & Wear track & 1 & $(\mathrm{SO} / \mathrm{SO} 04)^{2-}$ & 56 & 10 & 33 & n.d. & n.d. \\
\hline H-BED & Reference & $<1$ & $(\mathrm{SO3} / \mathrm{SO} 4)^{2-}$ & 66 & 7 & 19 & 7 & $<1$ \\
\hline H-BED & Wear track & $<1$ & $(\mathrm{SO3} / \mathrm{SO4})^{2-}$ & 63 & 14 & 22 & $<1$ & n.d. \\
\hline C-NAC-BED & Reference & $<1$ & $\mathrm{~S}-\mathrm{S} /(\mathrm{SO} 03 / \mathrm{SO} 4)^{2-}$ & 74 & 5 & 16 & 4 & 1 \\
\hline C-NAC-BED & Wear track & $<1$ & $(\mathrm{SO} / \mathrm{SO} 0)^{2-}$ & 61 & 12 & 26 & $<1$ & $<1$ \\
\hline
\end{tabular}

Notes: Values are rounded to whole numbers; it is indicated where less than $1 \%(<1)$ was detected and the value was below the detection limit (n.d.)

layer present. The distribution of carbon, oxygen and nitrogen atoms in the samples was characterized to confirm the chitosan immobilization observed in a similar study (Ashtiana et al., 2019)

The total $S$ amount remains unchanged within the experimental and measuring uncertainties on all samples. In detailed analyses, no disulfide (thio compounds) are detectable in the wear marks, only oxidation states of +4 are detectable. It is concluded that essentially one existing mucin layer was consumed in the tribo-test.

\section{Conclusions}

In this study, we have developed a cornea model for tribological testing of different types of eye drops. The presence of a mucin layer covering the top of the sample was verified using XPS, and changes in elemental composition following the tribological tests were detectable. Especially, disulphide bridges (S-S) could be uniquely proven on the surface of unworn C-NACBED samples. This indicates effectively bonding support of active substances in this solution. On all other tested systems, no disulphide bridges could be measured; however, $\mathrm{S}$ is present only in oxidized states, i.e. $\left(\mathrm{SO}_{3} / \mathrm{SO}_{4}\right)^{2-}$. The system is capable of identifying not only differences between the friction behavior (by means of $\mathrm{COF}$ ) of three different lubricant formulations compared to a control consisting of $0.9 \% \mathrm{NaCl}$ solution, but also showed the difference between C-NAC-BED and a placebo formulation containing non-thiolated chitosan. In addition to $\mathrm{COF}$ comparisons of the samples, the developed model is also sensitive to relatively small changes in Young's modulus. This observation is very important for the discussion focusing on DES (Efraim et al., 2020) or intraocular pressure changes that lead to changes in corneal stiffness (Liu and $\mathrm{He}$, 2009). In this study, C-NAC-BED revealed the lowest COF in both configurations with different Young's modi. For future studies, authors advise to perform long-term tribological tests that will take the drop application period into consideration and also the wear of the mucin layer covering the supporting gel.

\section{References}

Ashtiana, M.K., Zandi, M., Shokrollahi, P., Ehsani, M. and Baharvand, H. (2019), "Chitosan surface modified hydrogel as a therapeutic contact lens", Polymers for Advanced Technologies, Vol. 31 No. 4, pp. 741-748.

Cobb, J.A., Dunn, A.C., Kwon, J., Sarntinoranont, M., Sawyer, W.G. and Tran-Son-Tay, R. (2008), "A novel method for low load friction testing on living cells", Biotechnology Letters, Vol. 30 No. 5, pp. 801-806.

Colter, J., Wirostko, B. and Coats, B. (2017), "Coefficient of friction between carboxymethylated hyaluronic acid-based polymer films and the ocular surface", Investigative Ophthalmology \& Visual Science, Vol. 58 No. 14, pp. 6165-6174.

Cooper, B.G., Lawson, T.B., Snyder, B.D. and Grinstaff, M. W. (2017), "Reinforcement of articular cartilage with a tissue-interpenetrating polymer network reduces friction and modulates interstitial fluid load support", Osteoarthritis and Cartilage, Vol. 25 No. 7, pp. 1143-1149.

Dunn, A.C., Tichy, J.A., Urueña, J.M. and Sawyer, W.G. (2013b), "Lubrication regimes in contact lens wear during a blink”, Tribology International, Vol. 63, pp. 45-50.

Dunn, A.C., Urueña, J.M., Puig, E., Perez, V.L. and Sawyer, W.G. (2013a), "Friction coefficient measurement of an in vivo murine cornea", Tribology Letters, Vol. 49 No. 1, pp. 145-149.

Efraim, Y., Chen, F.-Y.T., Stashko, C., Cheong, K.N., Gaylord, E., McNamara, N. and Knox, S.M. (2020), "Alterations in corneal biomechanics underlie early stages of autoimmune-mediated dry eye disease", Fournal of Autoimmunity, Vol. 114, p. 102500.

Fischak, C., Klaus, R., Werkmeister, R.M., Hohenadl, C., Prinz, M., Schmetterer, L. and Garhöfer, G. (2017), "Effect of topically administered Chitosan-N-acetylcysteine on corneal wound healing in a rabbit model", Fournal of Ophthalmology, p. 6.

Jones, M.B., Fulford, G.R., Please, C.P., McElwain, D.L.S. and Collins, M.J. (2008), "Elastohydrodynamics of eyelid wiper”, Bulletin of Mathematical Biology, Vol. 70 No. 2, pp. 323-343.

Last, J.A., Liliensiek, S.J., Nealey, P.F. and Murphya, C.J. (2009), "Determining the mechanical properties of human corneal basement membranes with atomic force microscopy", Fournal of Structural Biology, Vol. 167 No. 1, pp. 19-24.

Last, J.A., Thomasy, S.M., Croasdale, C.R. and Russell, P. (2012), "Compliance profile of the human cornea as measured by an atomic force microscopy", Micron, Vol. 43 No. 12, pp. 1293-1298.

Liu, J. and He, X. (2009), "Corneal stiffness affects IOP elevation during rapid volume change in the eye", Investigative Opthalmology \& Visual Science, Vol. 50 No. 5, pp. 2224-2229.

Ludwig, A. (2005), "The use of mucoadhesive polymers in ocular drug delivery", Advanced Drug Delivery Reviews, Vol. 57 No. 11, pp. 1594-1639. 
Malamut, S., Kligerman, Y. and Etsion, I. (2009), "The effect of dwell time on the static friction in creeping elastic-plastic polymer spherical contact", Tribology Letters, Vol. 35 No. 3, pp. 159-170.

Mann, A. and Tighe, B.J. (2006), "Ocular biotribology and the contact lens: surface interactions and ocular response", Biomaterials and Regenerative Medicine on Ophthalmology, 2nd ed., pp. 45-74.

Molladavoodi, S., Kwon, H., Medley, J. and Gorbet, M. (2014), "Human corneal epithelial cell response to substrate stiffness", Acta Biomaterialia, Vol. 11, pp. 324-332.

Mollenhauer, O., Ahmed, S.I.-U., Spiller, F. and Haefke, H. (2006), "High-precision positioning and measurement systems for microtribotesting", Tribotest, Vol. 12 No. 3, pp. 189-199.

Ngai, V., Medley, J.B., Jones, L., Forrest, J. and Teichroeb, J. (2005), "Friction of contact lenses: silicone hydrogel versus conventional hydrogel", Life Cycle Tribology, pp. 371-379.

NIST (2012), "X-ray photoelectron spectroscopy database", Version 4.1, National Institute of Standards and Technology, Gaithersburg, available at: http://srdata.nist.gov/xps/

Raghunatan, V.K., Thomasy, S.M., Strøm, P., Yañez-Soto, B., Garland, S.P., Sermeno, J., Reilly, C.M. and Murphy, C.J. (2017), "Tissue and cellular biomechanics during corneal wound injury and repair", Acta Biomaterialia, Vol. 58, pp. 291-301.

Rennie, A.C., Dickrell, P.L. and Sawyer, W.G. (2005), "Friction coefficient of soft contact lenses: measurements and modelling", Tribology Letters, Vol. 18 No. 4, pp. 499-504.

Rico-del-Viejo, L., Lorente-Velázquez, A., HernándezVerdejo, J.L., García-Mata, R., Benítez-del-Castillo, J.M. and Madrid-Costa, D. (2017), "The effect of ageing on the ocular surface parameters", Contact Lens and Anterior Eye, Vol. 41 No. 1, pp. 5-12.

Samsom, M., Chan, A., Iwabuchi, Y., Subbaraman, L., Jones, L. and Schmidt, T.A. (2015), "In vitro friction testing of contact lenses and human ocular tissue: effect of proteoglycan (PRG4)", Tribology International, Vol. 89, pp. 27-33.

Shaw, A.J., Collins, M.J., Davis, B.A. and Carney, L.G. (2010), "Eyelid pressure and contact with the ocular surface", Investigative Opthalmology \& Visual Science, Vol. 51 No. 4, pp. 1911-1917.

Shih, P., Huang, C., Huang, T., Lin, H., Yen, J., Wang, I., Cao, H., Shih, W. and Dai, C. (2017), "Estimation of the corneal Youngs's modulus in vivo based on a fluid-filled spherical-shell model with", fournal of Ophthalmology, Vol. 2017, p. 11.

Ś ródka, W. (2011), "Evaluating the material parameters of the human cornea in a numerical model", Acta of Bioengineering and Biomechanics, Vol. 13 No. 3, pp. 77-85.

Sterner, O., Karageorgaki, C., Zürcher, M., Zürcher, S., Scales, C.W., Fadli, Z., Spencer, N.D. and Tosatti, S.G.P. (2017), "Reducing friction in the eye: a comparative study of lubrication by surface-anchored synthetic and natural ocular mucin analogues", ACS Applied Materials \& Interfaces, Vol. 9 No. 23, pp. 20150-20160.

Thomasy, S.M., Raghunathan, V.K., Winkler, M., Reilly, C. M., Sadeli, A.R., Russell, P., Jester, J.V. and Murphy, C.J. (2014), "Elastic modulus and collagen organization of the rabbit cornea: epithelium to endothelium", Acta Biomaterialia, Vol. 10 No. 2, pp. 785-791.

Thulasi, P. and Djalilian, A. (2017), "Update in current diagnostics and therapeutics of dry eye disease", Ophthalmology, Vol. 124 No. 11, pp. 27-33.

Tomala, A., Göçerler, H. and Gebeshuber, I.C. (2013), "Bridging nano- and microtribology in mechanical and biomolecular layers", Scanning Probe Microscopy in Nanoscience and Nanotechnology, Vol. 3, pp. 431-485.

Urueña, J.M., McGhee, E.O., Angelini, T.E., Dowson, D., Sawyer, G.S. and Pitenis, A.A. (2018), "Normal load scaling of friction in gemini hydrogels", Biotribology, Vol. 13, pp. 30-35.

Wu, J., Nasseri, M.A., Eder, M., Gavaldon, M.A., Lohmann, C.P. and Knoll, A. (2013), "The 3D eyeball FEA model with needle rotation", APCBEE Procedia, Vol. 7, pp. 4-10.

\section{Corresponding author}

Hakan Göcerler can be contacted at: hakan.gocerler@, tuwien.ac.at 